\title{
Evaluation of New Criteria for Diagnosis of Diabetes Mellitus Based on Follow-Up Study of Borderline Diabetes
}

\author{
Akira Ohneda, Takashi Kobayashi and Jiro Nihei \\ The Third Department of Internal Medicine, Tohoku \\ University School of Medicine, Sendai 980
}

\begin{abstract}
Ohneda, A., Kobayashi, T. and Nihei, J. Evaluation of New Criteria for Diagnosis of Diabetes Mellitus Based on Follow-Up Study of Borderline Diabetes. Tohoku J. exp. Med., 1982, 137 (4), 437-444 — In order to evaluate the validity of recently proposed criteria for diagnosis of diabetes mellitus, a retrospective study was performed in 315 patients with borderline diabetes, who were selected from approximately 4,000 outpatients under treatment for more than three years. They were divided into two groups, BI and BII, according to the 2 -hr blood glucose level in the $50 \mathrm{~g}$ glucose tolerance test $(\mathrm{GTT}): \mathrm{BI}$ between 100 and $135 \mathrm{mg} / 100 \mathrm{ml}$ and BII between 135 and $195 \mathrm{mg} / 100 \mathrm{ml}$. In group BI, glucose tolerance was deteriorated in 38 of 75 patients $(51 \%)$ and converted to diabetes mellitus in 21 patients $(28 \%)$. In group BII, 78 among 232 patients $(34 \%)$ developed diabetes mellitus. Furthermore, in $85 \%$ of the 99 cases of the conversion to diabetes mellitus from borderline diabetes, it occurred within 5 years. Diabetic retinopathy was observed in 25 of 72 patients $(35 \%)$ in group BI and in 60 of 212 patients $(28 \%)$ in group BII. More than two thirds of the patients with diabetic retinopathy observed in the borderline diabetes developed their retinopathy within 5 years. Normal insulin response during GTT was proved only in $21 \%$ and $17 \%$ among groups BI and BII, respectively. These results indicate that the patients with borderline diabetes, BI as well as BII, are closely related with diabetes mellitus, and that the patients with impaired glucose tolerance should be treated with diet restriction as in diabetes mellitus. - - borderline diabetes; diabetic retinopathy; glucose tolerance test; diagnostic criteria; insulin response
\end{abstract}

In the clinical fields of diabetes mellitus, it is absolutely important to make a correct diagnosis. Since 1959, several criteria for the diagnosis of diabetes mellitus have been proposed (Fajans and Conn 1959; Remein and Wilkerson 1961; Fitzgerald and Keen 1964; WHO Expert Committee 1965). In Japan, epidemiology and clinical research on diabetes mellitus have been performed on the basis of the proposed standard which was recommended in 1970 by the special committee of Japan Diabetes Society (Kuzuya et al. 1970). These criteria for the diagnosis of diabetes mellitus were founded from the mean values and the standard deviation of the blood glucose levels in the glucose tolerance test (GTT) among healthy people.

In 1979, however, the working group of National Institute of Health in USA (National Diabetes Data Group, NDDG 1979) and in 1980 the WHO Expert

Received for publication, January 7, 1982. 
Committee proposed new standards of GTT with $75 \mathrm{~g}$ glucose for diabetes. These criteria were settled from the long-term field works performed in the special areas (O'Sullivan and Mahan 1968; Keen et al. 1974; Bennett et al. 1976; Birmingham Diabetes Survey Working Party 1976; Jarrett et al. 1979). In these criteria, the cut-off points of blood glucose level in GTT were elevated in comparison with those proposed several years ago by various authors or organizations (Fajans and Conn 1959; Remein and Wilkerson 1961; Fitzgerald and Keen 1964; WHO Expert Committee 1965; Kuzuya et al. 1970).

An elevation of cut-off points of blood glucose in GTT results in a decrease of "false positive diabetes" on the one hand. However, this procedure may increase the cases of "false negative diabetes" on the other hand. Therefore, the validity of the new criteria of GTT proposed by NDDG or WHO remains to be investigated from various points. In this paper, a retrospective study was performed in the group of the so-called "borderline diabetes" in order to evaluate the new criteria for diabetes mellitus.

\section{Patients And Methods}

In this study the borderline diabetes was selected from the whole patients who were under treatment for more than three years in Diabetes Clinic of Tohoku University Hospital or three related hospitals (Municipal Hospitals of Sanuma of Miyagi Pref., Yumoto of Fukushima Pref. and Esashi of Iwate Pref.).

Since $50 \mathrm{~g}$ of glucose has been used for an oral GTT in our diabetes clinics, the following definition was made for the "borderline diabetes": Fasting blood glucose less than 120 $\mathrm{mg} / 100 \mathrm{ml}$, the peak level more than $160 \mathrm{mg} / 100 \mathrm{ml}$ and 2 -hr glucose level between 100 and $195 \mathrm{mg} / 100 \mathrm{ml}$. Furthermore, the borderline cases were divided into two groups, BI and BII, according to the 2 -hr blood glucose level; BI between 100 and $135 \mathrm{mg} / 100 \mathrm{ml}$ and BII between 135 and $195 \mathrm{mg} / 100 \mathrm{ml}$. The diagnosis of diabetes mellitus was made according to the new criteria proposed by WHO (1980), whereas the normal GTT was defined as follows: fasting blood glucose less than $120 \mathrm{mg} / 100 \mathrm{ml}$, the peak level less than $160 \mathrm{mg} /$ $100 \mathrm{ml}$ and 2 -hr level less than $100 \mathrm{mg} / 100 \mathrm{ml}$. These cut-off points of each group were calculated using the exchange formula proposed by Hakura (1980), who compared the blood glucose levels during $50 \mathrm{~g}$ GTT with those during $75 \mathrm{~g}$ GTT.

The numbers of patients of BI and BII were 79 and 236 , respectively, as shown in Table 1. These patients visited the diabetes clinics at various intervals for the clinical examinations, including ophthalmological investigation by expert ophthalmologists. GTT was performed in most of these patients for $120 \mathrm{~min}$ and blood samples were obtained at 30 min intervals, as reported previously (Ohneda et al. 1974). The changes in glucose tolerance were evaluated from GTT. Furthermore, in a small group of these patients, an insulin response in GTT was examined and was evaluated according to the criteria reported previously (Ohneda et al. 1977). In the present study, blood glucose implies the level of plasma glucose or capillary blood glucose and was measured by the glucose oxidase method

TABLE 1. Borderline diabetes selected in diabetic clinics

\begin{tabular}{clcccc} 
Group & & Male & Female & Total \\
\cline { 1 - 2 } University Hospital & BI & 17 & 12 & 29 \\
& BII & 71 & 28 & 99 \\
Related Hospitals & BI & 33 & 17 & 50 \\
& BII & 67 & 70 & 137
\end{tabular}


(Teller 1956). Plasma insulin was determined by the immunoassay using the double antibody system (Morgan and Lazarow 1963).

\section{Results}

The distribution of the age at the first visit is presented in Table 2 . In group BI, the numbers of patients aged 40 to 59 were $22(76 \%)$ and $30(60 \%)$ in the University Hospital and related hospitals, respectively. Similarly, in group BII, $56(57 \%)$ or $65(47 \%)$ patients were 40 to 59 years old when they visited their diabetes clinics for the first time. Therefore, the number of patients aged more than 40 in both BI and BII amounted to $89 \%$ in the study.

The changes in glucose tolerance are presented in Table 3 . In general, the percentage of patients who revealed deteriorated glucose tolerance was higher in the related hospitals than in University Hospital. In group BI, glucose tolerance improved in 4 to $28 \%$, remained unchanged in 36 to $40 \%$ and deteriorated in 32 to $60 \%$. In this group 21 patients $(28 \%)$ elicited diabetic glucose tolerance. In group BII, glucose tolerance was ameliorated in $33 \%$, remained unchanged in $34 \%$ and deteriorated in $34 \%$. Namely, 78 patients $(34 \%)$ of group BII revealed diabetic glucose tolerance. The cumulative occurrence rate of diabetes mellitus in the borderline cases is presented in Fig. 1. Here the occurrence of diabetes in groups BI and BII was separately calculated and figured out. In group BI, the cumulative incidence of diabetes increased in a course of duration, reaching $14 \%$ at the first year, $48 \%$ at the third year, and $76 \%$ at the fifth year. In contrast, that for group BII increased, amounting to $33 \%$ at the first year, $63 \%$ at the third year, and $87 \%$ at the fifth year. Therefore, 84 of 99 patients $(85 \%)$ with borderline

TABLE 2. Age at the first visit of borderline diabetics

\begin{tabular}{|c|c|c|c|c|c|c|}
\hline & Group & -39 & $40-49$ & $50-59$ & $60-69$ & $70-($ years $)$ \\
\hline \multirow[t]{2}{*}{ BI } & University Hospital & 2 & 12 & 10 & 3 & 0 \\
\hline & Related hospitals & 4 & 13 & 17 & 12 & 4 \\
\hline \multirow[t]{2}{*}{ BII } & University Hospital & 21 & 22 & 34 & 15 & 3 \\
\hline & Related hospitals & 9 & 14 & 51 & 42 & 21 \\
\hline
\end{tabular}

TABLE 3. Changes in glucose tolerance

Group Improved Unchanged Deteriorated Diabetic* Total

$\begin{array}{cccccc}\text { BI University Hospital } & 7 & 10 & 8 & 5 & 25 \\ & (28) & (40) & (32) & (20) & (100) \\ \text { Related hospitals } & 2 & 18 & 30 & 16 & 50 \\ & (4) & (36) & (60) & (32) & (100) \\ \text { BII University Hospital } & 41 & 39 & 16 & 16 & 96 \\ & (43) & (40) & (17) & (17) & (100) \\ & 35 & 39 & 62 & 62 & 136 \\ \text { Related hospitals } & (26) & (29) & (45) & (45) & (100)\end{array}$

* Patients showing deteriorated and diabetic glucose tolerance.

Numbers in parenthesis represent the percentages. 


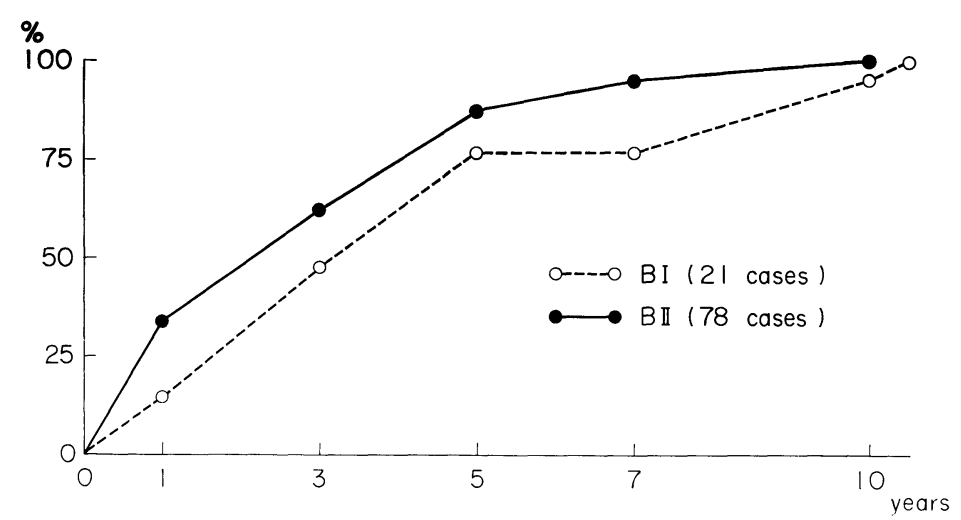

Fig. 1. Cumulative percentage of conversion to diabetes mellitus from borderline diabetes.

TABLE 4. Occurrence of diabetic retinopathy

\begin{tabular}{|c|c|c|c|c|c|}
\hline & \multirow{2}{*}{ Group } & \multicolumn{3}{|c|}{ Diabetic retinopathy } & \multirow{2}{*}{ Total } \\
\hline & & Present & Absent & Unproved & \\
\hline \multirow[t]{2}{*}{ BI } & University Hospital & $\begin{array}{c}7 \\
(27)\end{array}$ & $\begin{array}{c}19 \\
(73)\end{array}$ & 3 & 29 \\
\hline & Related hospitals & $\begin{array}{c}18 \\
(39)\end{array}$ & $\begin{array}{c}28 \\
(61)\end{array}$ & 4 & 50 \\
\hline \multirow[t]{2}{*}{ BII } & University Hospital & $\begin{array}{c}18 \\
(22)\end{array}$ & $\begin{array}{c}64 \\
(78)\end{array}$ & 17 & 99 \\
\hline & Related hospitals & $\begin{array}{c}42 \\
(32)\end{array}$ & $\begin{array}{c}88 \\
(68)\end{array}$ & 7 & 137 \\
\hline
\end{tabular}

Numbers in parenthesis represent the percentages against total patients excluding the unproved patients.

diabetes in groups BI and BII converted to those with diabetes mellitus. This figure implies the early development of diabetes mellitus among the patients with borderline glucose tolerance.

The development of diabetic retinopathy is presented in Table 4. To summarize, diabetic retinopathy was proved in 25 out of 72 patients $(35 \%)$ in group BI and in 60 out of $212(28 \%)$ in group BII, excluding the patients who were not examined by any ophthalmologists. Diabetic retinopathy seems to develop more frequently in group BI than in group BII and in the related hospitals than in University Hospital. However, no significant difference was observed in the rate of occurrence of diabetic retinopathy between these groups. The cumulative rate of the development into diabetic retinopathy in groups BI and BII is presented in Fig. 2. The cumulative incidence of diabetic retinopathy in 25 patients of group BI increased, reaching $32 \%$ at the first year, $48 \%$ at the third year and $68 \%$ at the fifth year. Similarly, that of diabetic retinopathy in 60 patients of group BII reached $42 \%$ at the first year, $72 \%$ at the third year and $90 \%$ at the fifth year. This picture implies that more than two thirds of diabetic retinopathy observed in borderline diabetes developed already by the fifth year. 


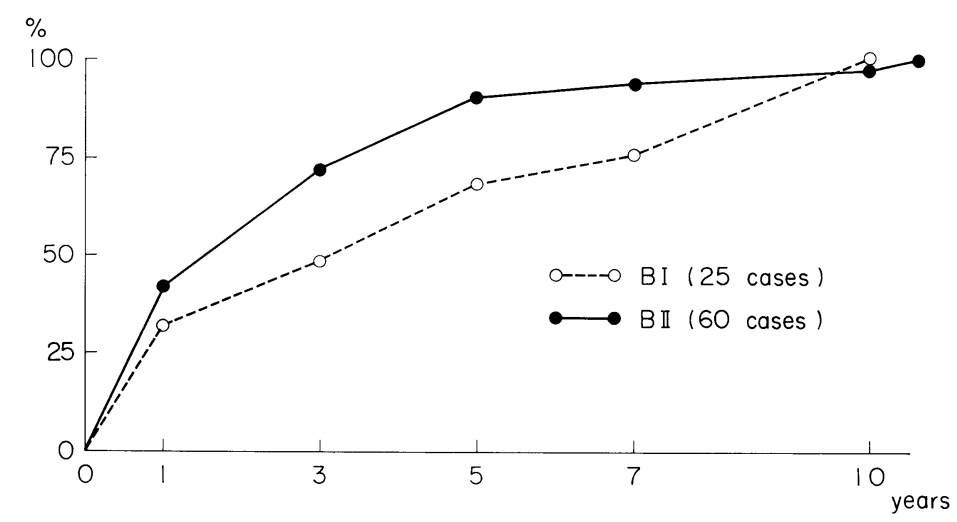

Fig. 2. Cumulative percentage of the occurrence of diabetic retinopathy in borderline diabetes.

TABLE 5. Insulin response in oral glucose tolerance test

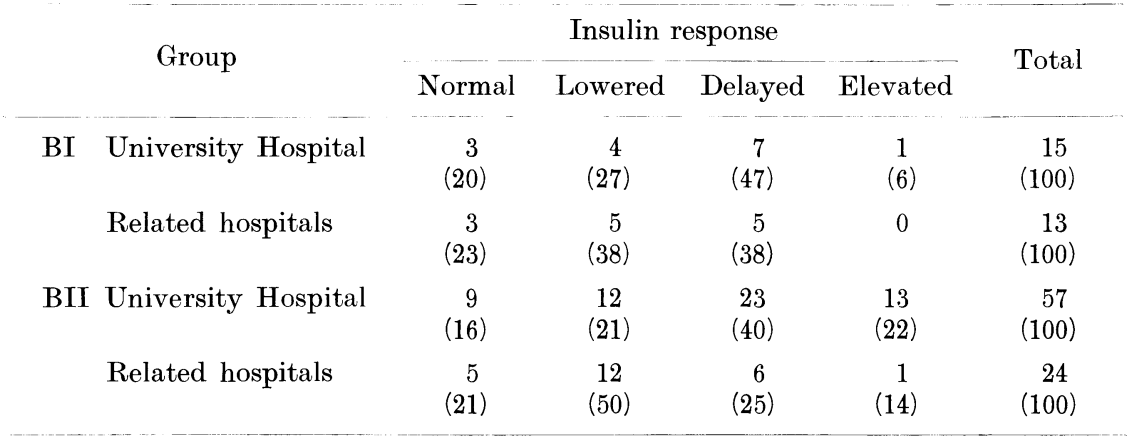

Numbers in parenthesis represent the percentages.

An insulin response in an oral GTT performed in 109 patients was presented in Table 5. Here, the insulin response was classified into several types according to the criteria reported previously (Ohneda et al. 1977). The normal response was observed only in $21 \%$ and $17 \%$ among groups BI and BII, respectively. In contrast, the lowered response or delayed response was observed in $75 \%$ of $\mathrm{BI}$ and $65 \%$ of BII.

\section{Discussion}

It is well understood that essentially the blood glucose levels, fasting or postprandial, distribute continuously, causing difficulties in separating diabetes mellitus from non-diabetics by GTT. In the previous criteria for the diagnosis of diabetes mellitus, the decision of the cut-off points was based on the mean and the standard deviation of the blood glucose levels obtained from healthy or nondiabetic population (Fajans and Conn 1959; Remain and Wilkerson 1961; Fitzgerald and Keen 1964; WHO Expert Committee 1965; Kuzuya et al. 1970). To the contrary, however, the cut-off line of the blood glucose level in the new criteria 
proposed by NDDG or WHO was drawn accoring to the results obtained from the long-term survey performed among the several population groups (O'Sullivan and Mahan 1968; Keen et al. 1974; Bennett et al. 1976; Birmingham Diabetes Survey Working Party 1976; Jarrett et al. 1979). Therefore, the procedure for the newly proposed criteria of diabetes mellitus does not differ from that for the previous standards of the disease, but the cut-off point was only elevated. As mentioned above, the changes in the criteria for the diagnosis of the disease give rise to serious influences in the clinical fields. Therefore, the validity of the new criteria should be investigated from the various points. The present study was performed in order to evaluate the new criteria by means of a follow-up study in the borderline diabetic groups. In this study, the borderline groups were divided into two groups, BI and BII, for the comparison of the criteria not only with diabetes mellitus but also with normal standard. Consequently, group BII corresponds to the group with impaired glucose tolerance (IGT) proposed by the new criteria, whereas group BI is included in the normal range of the new criteria and corresponds to the borderline diabetes in the previous Japanese standard (Kuzuya et al. 1970).

In the present study, diabetic retinopathy was frequently discovered during the follow-up period. Diabetic retinopathy was proved in 25 out of 72 patients $(35 \%)$ in group BI, while 60 out of 212 patients $(28 \%)$ in group BII. In the present study, microaneurysma of the retina was defined as one of the findings of diabetic retinopathy. It is well known that microaneurysma is observed in intoxication of carbon disulphide (Yamagata et al. 1966) and that the features of diabetic retinopathy are not unique to diabetes mellitus but are detected in other diseases (Kohner 1981). However, it is unlikely that most of the aneurysma detected in the present study is derived from such diseases other than diabetes. Therefore, the occurrence of retinal microaneurysma is regarded to be closely related with diabetes mellitus. Furthermore, it should be emphasized that in $80 \%$ of the patients with diabetic retinopathy, it developed within the early period less than 5 years after the onset of diabetes mellitus. These results are incompatible with the proposal that IGT is not a candidate for diabetes mellitus (NDDG 1979). According to the summary of NDDG, diabetic microangiopathy did not develop among the patients with chemical diabetes or borderline diabetes in the long-term studies. At present, the difference in the occurrence of diabetic retinopathy between their groups and ours cannot be fully interpreted. Differences in the subjects, general population or patients visiting hospitals, and in the method to detect the complications may be pointed out. However, if fluorescein angiography would have been employed, much higher incidence of diabetic retinopathy could be obtained, as Nielsen (1980) suggested.

In many cases of the present series, borderline diabetes was found to have converted to diabetes mellitus. That is, diabetic glucose tolerance was proved in 21 out of 75 patients (28\%) in group BI and in 78 out of 232 patiens (34 $\%$ ) in group BII. Furthermore, conversion to diabetes mellitus was observed in the 
early follow-up period, as shown in Fig. 1. The percentages of the development into diabetes mellitus within 5 years are $76 \%$ and $87 \%$ in groups BI and BII, respectively. According to NDDG (1979), the rate of development of diabetes mellitus among the groups of borderline diabetes is $1-5 \%$ per year. However, the mass survey performed in 1957 through 1962 in Japan indicated that the development of diabetes mellitus in general population was observed to increase at a rate less than $1 \%$ per year (Kosaka 1967). Therefore, the occurrence of diabetes in group BI as well as in group BII is demonstrated at a significantly elevated rate as compared with the general population $(p<0.001)$. These results also indicate the close relationship between borderline diabetes and diabetes mellitus.

In this study, an insulin response to oral glucose load was investigated in 99 patients. Only $20 \%$ of the patients revealed a normal pattern of insulin response, while $75 \%$ of them elicited an abnormal (lowered or delayed) response. The fact also supports the idea that borderline glucose tolerance (IGT and subdiabetic tolerance) is a prediabetic pattern and a potent candidate for diabetes. These results suggest the abnormality in the response of the pancreatic B cell to glucose even at the early stage of diabetes mellitus.

Recently, the effect of tolbutamide administration on the development of diabetes mellitus was investigated in a group with glucose intolerance (Sartor et al. 1980). According to their study, no individual developed diabetes mellitus in the group with sulfonylurea, while a higher incidence of diabetes mellitus was observed among the group without diet restriction $(29 \%$ ) or without sulfonylurea administration $(13 \%)$. In this context, a larger number of diabetes mellitus were observed in the group of impaired glucose tolerance without any treatment by Ito and her associate (1976), compared with the groups under control. The present study indicates that diabetes mellitus developed in a fairly large number of patients, even though they were treated with diet restriction. Therefore, from the present study it should be emphasized that the patients with impaired glucose tolerance have to be supervised under appropriate control for diabetes mellitus.

\section{Acknowledgments}

We express our thanks to Drs. F. Saito, Sanuma Hospital, M. Takeuchi, Yumoto Hospital and K. Kashiwagi, Esashi Hospital, for their cooperation in this study.

\section{References}

1) Bennett, P.H., Rushforth, N.B., Miller, M. \& LeCompte, P.M. (1976) Epidemiologic studies of diabetes in the Pima Indians. Recent Prog. Horm. Res., 32, 333-376.

2) Birmingham Diabetes Survey Working Party (1976) Ten-year follow-up report on Birmingham Diabetes Survey of 1061. Brit. med. J., 2, 35-37.

3) Fajans, S.S. \& Conn, J.W. (1959) The early recognition of diabetes mellitus. Ann. N.Y. Acad. Sci., 82, 208-218.

4) Fitzgerald, M.G. \& Keen, H. (1964) Diagnostic classification of diabetes. Brit. med. J., 1, 1568.

5) Hakura, R. (1980) Comparative study of $100 \mathrm{~g}, 75 \mathrm{~g}$ and $50 \mathrm{~g}$ glucose tolerance test. Igaku no Ayumi, 113, 709-714. (Japanese) 
6) Ito, U. \& Kawate, R. (1976) Development to overt diabetes from early diabetes. J. Jap. Diabet. Soc., 19, Suppl. 90. (Japanese)

7) Jarrett, R.J., Keen, H., Fuller, J.H. \& McCartney, M. (1979) Worsening to diabetes in men with impaired glucose tolerance. Diabetologia, 10, 25-30.

8) Keen, H.J., Jarrett, R.J. \& Fuller, J.H. (1974) Tolbutamide and arterial disease in borderline diabetics. In: Diabetes, edited by W.J. Mallaisse \& J. Pirart, Excerpta Medica, American Elsevier, pp. 588-602.

9) Kohner, E.M. (1981) Abnormal physiological process in the retina. In: Handbook of Diabetes Mellitus, edited by M. Brownlee, Garland STPM Press, New York-London, Vol. 4, pp. 3-21.

10) Kosaka, K. (1967) Follow-up study on diagnosis of diabetes mellitus by carbohydrate rich meal test. J. Jap. Diabet. Soc., 10, 229-231. (Japanese)

11) Kuzuya, N. \& Committee for Diagnosis of Diabetes Mellitus (1970) Report from the special committee for diabetes mellitus using glucose tolerance test. J. Jap. Diabet. Soc., 13, 1-7. (Japanese)

12) Morgan, C.R. \& Lazarow, A. (1963) Immunoassay of insulin: two antibody system. Diabetes, 12, 115-126.

13) National Diabetes Data Group (1979) Classification of diabetes mellitus and other categories of glucose intolerance. Diabetes, 28, 1039-1057.

14) Nielsen, N.V. (1980) Fluorescein angiography in persons with slightly abnormal glucose tolerance. Acta endocr., 94, Suppl. 238, 77-84.

15) Ohneda, A., Sato, M. \& Yamagata, S. (1974) Insulin response to tolbutamide-glucose load in normal and diabetic subjects. Tohoku J. exp. Med., 114, 171-182.

16) Ohneda, A., Watanabe, K., Maruhama, Y., Itabashi, H., Horigome, K., Chiba, M., Kai, Y., Sakai, T. \& Okuguchi, F. (1977) Changes in glucose tolerance and insulin response in subclinical diabetes after treatment. Tohoku J. exp. Med., 122, 199-207.

17) O'Sullivan, J.B. \& Mahan, C.M. (1968) Prospective study of 352 young patients with chemical diabetes. New Engl. J. Med., 273, 1038-1041.

18) Remein, Q.R. \& Wilkerson, H.L.C. (1961) The efficiency of screening test for diabetes. J. chronic Dis., 13, 6-21.

19) Sartor, G., Schersten, B., Carlström S, Melander, A., Nordén, A. \& Persson, G. (1980) Ten-year follow-up of subjects with impaired glucose tolerance. Prevention of diabetes by tolbutamide and diet regulation. Diabetes, 29, 41-49.

20) Teller, J.D. (1956) Direct, quantitative, colorimetric determination of serum or plasma glucose. In: Abstracts of Papers, 130th Meeting, Amer. Chem. Soc., Sept. p. $69 \mathrm{c}$.

21) WHO Expert Committee on Diabetes Mellitus (1965) WHO Tech. Rep., Ser. 310, 715.

22) WHO Expert Committee on Diabetes Mellitus (1980) WHO Tech. Rep., Second Report, Ser. 646, 7-14.

23) Yamagata, Y., Nemoto, T., Takahashi, M., Yada, A., Suzuki, K, Tsuchida, H., Saito, K. \& Kusunoki, N. (1966) Carbon disulphide nephrosclerosis. II. A clinicopathological study based on renal biopsy materials. J. Jap. Diabet. Soc., 9, 218-225. (Japanese) 